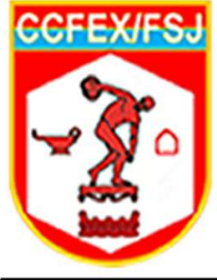

Revista de Educação Física

Joumal of Physical Education

Some page: : unn revistadeeduoacaotisioa.00m

Artigo Original

Original Article

\title{
Comparação dos níveis de agilidade em crianças em idade escolar praticantes e não praticantes de judô: um estudo seccional
}

\section{Comparison of Agility Levels in Judo-Practicing and Non-Practicing School- Age Children: A Cross-Sectional Study}

Wescley Rufino de Queiroz, Bacharel ${ }^{1}$; Rodrigo Gomes de Souza Vale ${ }^{1,2,3,4} \mathrm{PhD}$; Leandro de Lima e Silva ${ }^{2} \mathrm{MS}^{1,2}$ Carlos Soares Pernambuco ${ }^{1}$ PhD; Rodolfo Alkmim Moreira Nunes ${ }^{2,3}$ PhD; Ignácio Antônio Seixas-da-Silva ${ }^{1,2,3}$ MS

Recebido em: 10 de setembro de 2019. Aceito em: 11 de outubro de 2019.

Publicado online em: 31 de outubro de 2019.

\section{Resumo}

Introdução: A agilidade é fundamental para o judô, uma luta que demanda força e potência e utiliza mudanças bruscas de direção. Pouco se investigou, no Brasil, a respeito da qualidade física agilidade em crianças escolares pré-puberes.

Objetivo: Comparar o nível de agilidade entre crianças praticantes e não-praticantes de judô de uma escola pública.

Métodos: Pesquisa descritiva de corte transversal comparativa, da qual participaram 77 crianças de ambos os sexos: 47 praticantes de judô e 30 não praticantes. Teste $t$ de Student para amostras independentes foi aplicado para a comparação entre os grupos.

Resultados: Os praticantes regulares de judô exibiram maiores níveis de agilidade $(10,98 \pm 0,79$ segundos $)$ do que os não praticantes de judô $(13,48 \pm 0,74$ segundos) e a diferença foi significativa $(\mathrm{p}=0,0001)$.

Conclusão: Crianças que praticam judô apresentaram níveis maiores de agilidade do que as participantes de iniciação esportiva em outras modalidades, sugerindo que o treinamento de judô pode favorecer o desenvolvimento dessa capacidade física mais do que as

Pontos-Chave Destaque

- Poucos estudos avaliaram agilidade de escolares prépuberes.

- Judocas de ambos os sexos apresentaram níveis maiores de agilidade do que as crianças que praticavam outras modalidades.

- Há necessidade de mais estudos quanto a valências físicas nesse tipo de população. demais modalidades. Outros estudos de intervenção devem ser conduzidos para esclarecer essa associação.

Palavras-chave: artes marciais, aptidão física, crianças, qualidades físicas.

\begin{abstract}
Introduction: Introduction: Agility is fundamental to judo, a fight that demands strength and power and uses sudden changes of direction. Little has been investigated in Brazil regarding physical quality agility in prepubescent school children.

Objective: To compare the level of agility between practicing and non-practicing children of judo in a public school.

Methods: A descriptive comparative cross-sectional study was conducted in which 77 children of both sexes participated: 47 judo practitioners and 30 non-practitioners. Student's t-test for independent samples was applied for comparison between groups.
\end{abstract}

$\S$ Autor correspondente: Leandro de Lima e Silva - e-mail: I.limaesilva@gmail.com

Afiliações: ${ }^{1}$ Universidade Estácio de Sá (Campus Cabo Frio); ${ }^{2}$ Universidade do Estado do Rio de Janeiro (PPGCEE/UERJ);

${ }^{3}$ Laboratório do Exercício e Esporte (LABES/UERJ); ${ }^{4}$ Bolsista de pesquisa Produtividade UNESA. 
Results: Regular judo practitioners exhibited higher levels of agility (10.98 \pm 0.79 seconds) than non-judo practitioners $(13.48 \pm 0.74$ seconds) and the difference was significant ( $\mathrm{p}=0.0001)$.

Conclusion: Children who practice judo showed more agility than participants of sports initiation in other modalities suggesting that judo training may favor the development of this physical capacity more than the other modalities. Further intervention studies should be conducted to clarify this association.

Keywords: martial arts, physical fitness, children, physical qualities.

\section{Keypoints}

- Few studies have evaluated agility of prepubertal schoolchildren.

- Judokas of both sexes presented higher levels of agility than children who practiced other modalities.

- There is a need for further studies on physical valences in this type of population.

\section{Comparação dos níveis de agilidade em crianças em idade escolar praticantes e não praticantes de judô: um estudo seccional}

\section{Introdução}

O judô foi criado por Jigoro Kano no Japão em 1882 e é baseado em outra arte marcial japonesa, o jiu-jitsu.Este se fundamenta em princípios benéficos à integridade física $\mathrm{e}$ moral dos praticantes sempre priorizando o respeito e o bom comportamento tanto nos tatames como fora deles(1). Também conhecido como o caminho suave, o judô é caracterizado por ser uma arte marcial que desenvolve autoconfiança, tranquilidade mental, agilidade e que exige velocidade de reação, coordenação neuromuscular, força explosiva de pernas e braços, equilíbrio dinâmico e recuperado, e flexibilidade de tronco e pernas $(2,3)$. estando classificada dentre as modalidades de esportes de combate como uma luta de percussão ou domínio(2). O judô possui movimentos muito específicos, o que requer do praticante uma demanda metabólica elevada por caracterizar-se em movimentos de curta duração e alta intensidade(4). A potência e a agilidade no momento de desencadear um golpe são de grande importância para a vitória ou derrota do judoca no momento da luta, quanto mais ágil e potente for o judoca, maior sua probabilidade de vitória(5).

A modalidade apresenta bons motivos para sua implantação no contexto escolar, pois atua em diversos aspectos dentre eles: desenvolvimento motor, valores físicos e principalmente a aprendizagem de valores educacionais(6). E contribui para promover o desenvolvimento da criança em sua plenitude, considerando os valores da arte marcial transmitidos, seja no desenvolvimento das habilidades motoras, no aspecto cognitivo ou no contexto social, estão relacionados à melhora do rendimento no âmbito escolar $(7,8)$.

A agilidade é um termo que se refere à capacidade do atleta de mudar de direção de forma rápida e eficaz. É a manifestação da velocidade de forma acíclica(9). Para Barbanti(8), a agilidade é fundamental, pelo fato de ser uma luta explosiva a qual necessita de mudanças bruscas de direção, sem perder velocidade, o equilíbrio e o controle do adversário, sendo uma das valências físicas mais relevantes tanto em esportes coletivos como em individuais(10), pois, relaciona-se diretamente ao desenvolvimento de outras: força, velocidade, flexibilidade e coordenação(9). O conjunto dessas valências é imprescindível o desempenho físico(11). A literatura mostra que esportes de artes marciais promovem tais qualidades físicas nas crianças e, ainda, podem melhorar a autoconfiança e a autoestima(1). Pouco se investigou, no Brasil, a respeito da qualidade física agilidade em crianças escolares pré-puberes. 
O objetivo do presente estudo foi comparar o nível de agilidade entre crianças praticantes e não-praticantes de judô de uma escola públicas da cidade de Araruama/RJ.

\section{Métodos}

\section{Desenho de estudo e amostra}

O presente estudo trata-se de uma pesquisa descritiva de corte transversal comparativa(12), realizada na cidade São Vicente, Araruama / RJ, em que o universo é composto por crianças oriundas de família com baixo poder aquisitivo em fase escolar da rede pública de ensino, do interior do Estado do Rio de Janeiro.

Os critérios de escolha para a inclusão dos voluntários no estudo tiveram como prérequisito ter idade entre 8 e 10 anos; ter no mínimo 1 ano de prática de judô e estarem estudando no Ensino Fundamental I.

Os critérios de escolha para exclusão dos voluntários no estudo tiveram como prérequisito não ser assíduo aos treinos ou não ter liberação médica para a prática.

Após o término do processo de amostragem, a amostra foi composta por 77 crianças: 47 praticantes de judô e 30 não praticantes, entre meninos e meninas.

\section{Aspectos éticos}

A pesquisa foi realizada dentro das normas éticas prescritas na Resolução CNS n ${ }^{\circ} 466$, de 12 de dezembro de 2012, do Conselho Nacional de Saúde, que norteia a pesquisa envolvendo seres humanos.

\section{Agilidade}

Agilidade foi a variável desfecho do estudo. Para avalia-la utilizou-se o teste de Shuttle run(13) que estima o tempo (em segundos) necessário para que o indivíduo complete a tarefa proposta. Assim, quanto maior o tempo gasto, maior o nível de agilidade.

\section{Medidas antropométricas}

Para a caracterização da amostra foram tomadas as medidas antropométricas massa corporal $(\mathrm{kg})$ e estatura $(\mathrm{m})$, mensuradas por meio de balança com estadiômetro da marca Filizola ${ }^{\circledR}$. Para a aferição utilizou-se o protocolo da International Society for the Advancement of Kinanthropometry(14) e foi calculado o índice de massa corporal (IMC).

\section{Procedimentos de coleta de dados}

A tarefa do Shuttle run(13) consistiu em transferir os dois blocos de um lado para outro da seguinte forma: partindo da primeira linha o indivíduo correu imprimindo sua maior velocidade até a linha de referência pegou um dos blocos (passando ao menos um dos pés sobre a linha) e volta para a linha de chegada para colocar o bloco do outro lado da linha (o bloco não poderia ser atirado). Terminada esta etapa o mesmo procedimento foi feito com o segundo e último bloco. Os materiais utilizados foram dois blocos de madeira $(5 \mathrm{~cm}$ x $5 \mathrm{~cm} \times 10 \mathrm{~cm}$ ), que ficaram posicionados $10 \mathrm{~cm}$ após a linha de referência com distância de $30 \mathrm{~cm}$ entre eles, e um cronômetro (uso do avaliador). A amostra teve a oportunidade de se familiarizar previamente com o teste.

O teste teve início com o comando do avaliador para iniciar a operação de corrida, e terminou quando o avaliado deixou o segundo bloco no chão e passou com ao menos um dos pés da linha de partida novamente. $O$ resultado do teste será o tempo gasto pelo avaliado para realizar este procedimento. Foram realizadas duas tentativas, e foi utilizado o menor resultado obtido. Depois do pré-teste o atleta teve 20 minutos de recuperação, para retornar a homeostase e após realizar as atividades do grupo e pós-teste, onde sorteio dos grupos foi realizado após a aplicação do teste Shuttle run.

Os dois grupos participam regularmente das aulas de educação física na escola, 2 vezes por semana totalizando uma hora e meia de atividade semanal, em que são trabalhadas as iniciações aos principais desportos, jogos e brincadeiras.

O grupo de alunos que pratica judô, além das aulas de educação física, faz em todas as aulas um aquecimento em circuito, treina amortecimentos de quedas (ukemi), técnicas especificas da luta, como movimentação de quadril, braços e pernas, com velocidade e força. Os praticantes treinam repetidas vezes técnicas de projeção, como: ippon seoi nage, ouchi gari, osoto gari, de ashi barai, ogoshi, entre outras. Fazem o "randori", treinamento livre, a luta propriamente dita, em que o objetivo de derrubar o oponente de costas no tatame e/ou imobilizar, segurando-os de costas por 20 segundos. Os estrangulamentos e 
chaves de articulações não são praticados nesta faixa etária.

\section{Análise estatística}

Os dados foram analisados no Excel® 2007, e apresentados como média, desvio padrão e valores mínimos e máximos. Teste $t$ de Student para amostras independentes foi aplicado para a comparação entre os grupos. O nível de significância estatística considerado foi $p<0,05$.

\section{Resultados}

O estudo foi realizado com uma amostra de 77 alunos, de ambos os sexos, que se caracte- rizou por apresentar média de idade de 9 anos, média de altura de144 $\mathrm{cm}$ e média de massa corporal de 33,21 quilogramas.

A Tabela 1 apresenta as características da amostra, dividida nos dois grupos analisados no estudo (não praticantes de judô; e praticantes de judô), na qual se observa a massa corporal, estatura, idade e resultado no teste para a verificação dos níveis de agilidade Shuttle run. Os praticantes de judô, embora mais pesados, exibiram nível maior de agilidade (tempo menor gasto na realização da tarefa) $(10,98 \pm 0,79 \mathrm{seg})$ do que os não praticantes de judô $(13,48 \pm 0,74 \mathrm{seg})$. O teste $t$ mostrou que essa diferença foi significativa $(p=0,0001)$.

Tabela 1 - Características da amostra e resultados do teste de agilidade (Shuttle run) em crianças $(\mathrm{N}=77)$

\begin{tabular}{lcccccccc}
\hline Característica & \multicolumn{3}{c}{ Não Praticantes de Judô $(\mathbf{n}=\mathbf{3 0})$} & \multicolumn{3}{c}{ Praticantes de Judô (n=47) } \\
\cline { 2 - 9 } & Média & DP & Min. & Max. & Média & DP & Min. & Max. \\
\hline \hline Massa corporal (Kg) & 35,31 & 8,08 & 24,00 & 55,40 & 42,91 & 10,09 & 25,00 & 61,07 \\
Estatura (m) & 1,32 & 0,11 & 1,10 & 1,50 & 1,43 & 0,13 & 1,10 & 1,60 \\
Idade (anos) & 9,20 & 0,72 & 8,00 & 10,00 & 9,36 & 0,89 & 8,00 & 10,00 \\
Shuttle run (seg) & 13,48 & 0,74 & 12,00 & 14,90 & 10,98 & 0,79 & 9,80 & 12,70 \\
\hline
\end{tabular}

Kg: quilogramas; m: metro; seg: segundos; DP: desvio padrão; Min: valor mínimo; Max: valor máximo.

\section{Discussão}

O principal achado do presente estudo foi que crianças praticantes de judô há mais de um ano apresentaram maior nível de agilidade do que as não praticantes, embora exibissem maior massa corporal (peso). Não foi identificado nenhum estudo que tivesse utilizado a mesma metodologia em crianças escolares com idades entre 9 e 10 anos. Observa-se que a falta de padronização nos métodos de avaliação impossibilita a comparação inter estudos.

Em um estudo realizado com crianças e adolescentes com faixa etária entre 12 a 16 anos Krebs e Macedo(15) observaram que os meninos são mais ágeis que as meninas e que, quanto maior a idade, maior o nível de agilidade, isto é, menor é tempo no teste. No presente estudo, observou-se que os participantes praticantes regulares de judô apresentaram média de idade ligeiramente maior do que os não praticantes de judô. Todavia, não foi examinada a associação de idade com agilidade nem se houve diferença segundo sexo.

Um estudo em judocas, demonstrou que a agilidade somada a velocidade contribui para execução de ações na luta. Sua pesquisa traçou o perfil físico dos atletas judocas, através da bateria de teste do Proesp-BR, sendo que a agilidade dos participantes classificou-se dentro dos parâmetros considerados bons(10).

O judô apresenta uma forma particular e muito específica de treinamento(16). Os autores afirmaram que, geralmente, não existe um trabalho diferenciado para os atletas das várias faixas etárias que treinam esta modalidade e que a capacidade de um indivíduo de responder ao treinamento começa a acentuar a partir da puberdade. Deixando claro que não se pode obter ganhos significativos na agilidade por crianças na idade dos participantes do presente estudo, tendo em vista que a idade média para a puberdade é entre 12 e 13 anos. À medida que se pratica uma determinada habilidade, com o passar do tempo, o indivíduo torna-se mais 
habilidoso nos padrões de movimento dos membros e do corpo, e assim, tentando adaptar esse padrão a novos contextos ambientais(17). No presente trabalho, foram avaliados os níveis de agilidade em crianças experientes no judô, o que permite inferir que a constância do treinamento pode ter contribuído para níveis maiores de agilidade. Bortoni et. al(18), em estudo experimental demonstrou que duas sessões semanais de treinamento em iniciação esportiva, de diversas modalidades, promoveram melhora significativa na agilidade (avaliada pelo teste Shutlle Run), assim, a iniciação esportiva é recomendada para favorecer o pleno desenvolvimento de crianças e adolescentes.

É importante que sejam conduzidos outros estudos quanto à valência física agilidade, pois, a literatura é escassa em crianças brasileiras. A recomendação justifica-se pelo fato de que a agilidade está diretamente ligada ao desempenho motor, maturação biológica, composição corporal e experiência de prática esportiva(19). Além disso, há necessidade de se estabelecer uma padronização nos testes para que seja possível a comparação entre os estudos.

\section{Pontos fortes e limitações do estudo}

O ponto forte deste estudo foi examinar os níveis de agilidade entre crianças praticantes e não praticantes de judô em fase escolar, pois, estudos científicos nesse tipo de população no Brasil, e no município de São Vicente/RJ, são escassos.

A principal limitação do estudo foi a amostra que por ser de conveniência, abrangeu somente uma escola, não sendo representativa de todas as crianças em idade escolar no município.

Outra limitação foi que não se trata de estudo de intervenção, por conseguinte só foi possível examinar associação sem possibilidade de serem feitas inferências causais.

Finalmente, houve limitações quanto às análises de associação - não foram feitas quanto a relação de nível de agilidade com idade nem segundo sexo. Quanto à variável sexo, a diferença estatística encontrada em agilidade entre as crianças que praticavam e as que não praticavam judô, indica a robustez do achado, uma vez que, de acordo com a literatura meninos são mais ágeis que meninas e tal separação não foi feita, o que poderia enfraquecer a associação. Quanto à idade, a variação foi menor do que $10 \%$, dessa forma, qualquer análise de associação, provavelmente, não detectaria diferenças face à homogeneidade dos dados.

\section{Conclusão}

O presente estudo propôs-se a verificar os níveis de agilidade de crianças praticantes e não praticantes de judô. Observou-se que os voluntários praticantes regulares de judô, há mais de um ano, tiveram um menor tempo, no teste de verificação de agilidade Shuttle run, caracterizando um melhor nível de agilidade.

Conclui-se que o treinamento de judô por crianças em fase escolar pode ser uma estratégia para melhorar os níveis da agilidade. Estudos de intervenção devem ser conduzidos para esclarecer essa associação.

Sugere-se, ainda, a continuidade nas pesquisas sobre o assunto controlando os níveis de intensidade e volume, assim como as atividades nas aulas de educação física escolar.

\section{Declaração de conflito de interesses}

Não há nenhum conflito de interesses em relação ao presente estudo.

\section{Declaração de financiamento}

Este estudo não contou com nenhum financiamento.

\section{Referências}

1. Laux RC, Zanini D. Identidade antropométrica de praticantes de judô de alto rendimento entre 11 e 17 anos do Município de Chapecó-SC. Biosaúde. 2016;16(2): 45-51.

2. Rosa CAF de L. Treinamento desportivo carga, estrutura e planejamento. São Paulo: Phorte; 2001. 140 p.

3. De Araújo. Judô: da história à pedagogia do esporte. Monografia apresentada à Faculdade de Educação Física da Universidade Federal de Goiás. Goiânia, GO, 2005.

4. Nunes AV, Rubio K. As origens do judô brasileiro: a árvore genealógica dos medalhistas olímpicos. Revista Brasileira 
de Educação Física e Esporte. [Online] 2012;26(4): 667-678. Available from: doi:10.1590/S1807-55092012000400011

5. Fabrini SP, Brito CJ, Mendes EL, Sabarense CM, Marins JCB, Franchini E. Práticas de redução de massa corporal em judocas nos períodos pré-competitivos. Revista Brasileira de Educação Física e Esporte. [Online] 2010;24(2): 165-177. Available from: doi:10.1590/S180755092010000200002

6. Baptista CF dos S. Judô: da escola à competição. Edição: 3a. Rio de Janeiro, RJ: Sprint; 1999.

7. Schmidt RA, Wrisberg CA. Aprendizagem e performance motora: uma abordagem da aprendizagem baseada no problema. Porto Alegre, RS: ArtMed; 2001. 352 p.

8. Barbanti VJ. Dicionário de educação fisica e esporte. Edição: Edição revista e ampliada. Barueri, SP, Brasil: Editora Manole; 2011.

9. Sheppard JM, Young WB. Agility literature review: classifications, training and testing. Journal of Sports Sciences. [Online] 2006;24(9): 919-932. Available from: doi:10.1080/02640410500457109

10. Preux C. Perfil da Aptidão física de praticantes de Judô do Centro Universitário do Leste de Minas Gerais. Revista Digital de Educação FísicaMovimentum.2006;1(2):1-17.Disponível em:https://www.unileste.edu.br/movime ntum/Artigos_V1N1_em_pdf/moviment um_preux_cristiano.pdf [Accessed: 29th October 2019].

11. Rebelo AN, Oliveira J. Relação entre a velocidade, a agilidade e a potência muscular de futebolistas profissionais. Revista Portuguesa de Ciências do Desporto. 2006;6(3): 342-348.
12. Thomas JR, Nelson JK, Silverman SJ. Métodos de Pesquisa em Atividade Física. Edição: 6. Porto Alegre, RS: Artmed; 2012.

13. Johnson BL, Nelson JK. Practical Measurements for Evaluation in Physical Education. Subsequent edition. New York: Macmillan Pub Co; 1986. 475 p.

14. Marfell-Jones TOAS and LCM, Stewart A, Marfell-Jones M, Kinanthropometry IS for A of. International Standards for Anthropometric Assessment. Glaskow, Scotland: International Society for the Advancement of Kinanthropometry; 2011. 137 p.

15. Krebs RJ, Macedo FO. Desempenho da aptidão física de crianças e adolescentes. Revista Digital, Buenos Aires. 2005;85(6):1-1 [Online] Available from: https://www.efdeportes.com/efd85/aptid ao.htm [Accessed: 29th October 2019]

16. Amorim AR, Drigo A, Molina R. Judô na adolescência: capacidade aeróbia e anaeróbia, composição corporal $e$ treinamento. Monografia Bacharelado em Educacçao Fisica, Deparmento de Educacçao Fisica do Instituto de Biocencias da UNSEP-Campus Rio Claro. 2001. Available from: https://novaescola.org.br/conteudo/1229/ o-lugar-da-luta-nas-aulas-de-educacaofisica [Accessed: 29th October 2019]

17. Magill RA. Aprendizagem Motora: Conceitos e Aplicações. Edição: 1a. São Paulo: Blucher; 2000.

18. Bortoni WL, Bojikian LP. Crescimento e aptidão física em escolares do sexo masculino, participantes de programa de iniciação esportiva. Brazilian Journal of Biomotricity. 2007; 1:114-22. [Online] Available from: http://www.scielo.br/scielo.php?script=s ci_nlinks\&ref $=000085 \&$ pid $=$ S0101 $3289201000030001400002 \& \operatorname{lng}=\mathrm{pt}$ [Accessed: 29th October 2019] 
910 Rev Ed Física / J Phys Ed - Agilidade em crianças praticantes e não praticantes de judô

19. Bojikian LP, Teixeira CP, Böhme MTS, Ré AHN. Relações entre crescimento, desempenho motor, maturação biológica e idade cronológica em jovens do sexo masculino. Revista Brasileira de Educação Física e Esporte. [Online] 2005;19(2): 153-162. Available from: doi:10.1590/S1807-55092005000200006 